\title{
Estimating gambling venue catchments for impact assessment using a calibrated gravity model
}

\author{
Francis Markham ${ }^{1}$, Bruce Doran ${ }^{2}$ and Martin Young ${ }^{3}$
}

Menzies School of Health Research, Darwin, NT, Australia ${ }^{1}$ - Fenner School of Environment and Society, The Australian National University, Canberra, ACT, Australia ${ }^{2}$ - School of Tourism and Hospitality Management, Southern Cross University, Coffs Harbour, NSW, Australia ${ }^{3}$

Correspondence to: Francis Markham, Fenner School of Environment and Society, The Australian National University, Building 141, Linnaeus Way, Canberra, ACT 0200, Australia. E-mail: francis.markham@anu.edu.au

This is an Accepted Manuscript of an article published by Taylor \& Francis Group in International Journal of Geographical Information Science on 1 October 2013, available online: http://dx.doi.org/10.1080/13658816.2013.838770

This work should be cited as:

Markham, F., Doran, B., \& Young, M. (2014). Estimating gambling venue catchments for impact assessment using a calibrated gravity model. International Journal of Geographical Information Science, 28(2), 326-342. doi:10.1080/13658816.2013.838770

\begin{abstract}
Gambling using electronic gaming machines has emerged as a significant public health issue. While social impact assessments are required prior to the granting of new gaming licenses in Australia, there are few established techniques for estimating the spatial distribution of a venue's clientele. To this end, we calibrated a Huff model of gambling venue catchments based on a geocoded postal survey $(n=7,040)$. We investigated the impact of different venue attractiveness measures, distance measures, distance decay functions, levels of spatial aggregation, and venue types on model fit and results. We then compared model estimates for different behavioural subgroups. Our calibrated spatial model is a significant improvement on previously published models, increasing $R^{2}$ from 0.23 to 0.64 . Venue catchments differ radically in size and intensity. As different population subgroups are attracted to different venues, there is no single best index of venue attractiveness applicable to all subpopulations. The calibrated Huff model represents a useful regulatory tool for predicting the extent and composition of gambling venue catchments. It may assist in decision making with regard to new license applications and evaluating the impact of health interventions such as mandated reductions in EGM numbers. Our calibrated parameters may be used to improve model accuracy in other jurisdictions.
\end{abstract}




\section{Background}

Gambling is a significant public health issue wherever commercial gambling opportunities are widely available. Estimates of gambling-related harm in the general population of Western countries range from 1.8\% in Australia (Productivity Commission, 1999) to $7.8 \%$ in Canada (Currie et al., 2006) depending on the measure used. At the level of the individual, the harms associated with gambling may include psychiatric problems, suicide, alcohol and drug problems, financial problems, and criminal behavior (Korn \& Shaffer, 1999). Other gambling-related harms such as regressive distribution of economic resources are social determinants of health and wellbeing that operate at the community level (Productivity Commission, 1999).

Gambling liberalisation during the last thirty years has resulted in the proliferation of commercial gambling opportunities in many developed countries. In the United States, for example, the number of states that authorise casino gambling rose from two in 1988 to thirty-eight in 2011 (American Gaming Association, 2012; Eadington, 1998). Similarly, in Australia, the number of electronic gaming machines (EGMs, the Australian variant of the slot machine) increased from 48,439 to 198,725 in the thirty years to 2010 (Office of Economic and Statistical Research, 2012; Wilkinson, 1996).

Increased gambling accessibility has resulted in a rise in the prevalence of gambling-related harms (Shaffer, Hall, \& Bilt, 1999; Storer, Abbott, \& Stubbs, 2010). In Australia, EGM density has been closely associated with elevated rates of gambling harm (Productivity Commission, 1999). When considered at the scale of the state jurisdiction, the availability of EGMs in venues other than casinos was associated with a tripling of the prevalence of problem gambling (Productivity Commission, 1999), with each additional 100 EGMs associated with 79 new problem gamblers (Storer et al., 2010).

Given that EGMs are a venue-based form of gambling, opportunities exist to intervene at the venue level to reduce EGM-related harm. While reversing the trend of increased gambling accessibility is politically challenging, other venue-level interventions such as self-exclusion programs, limits on access to automatic teller machines, and caps on machine numbers in venues, have become routine. In particular, every jurisdiction in Australia mandates that social impact assessments be undertaken prior to the granting of a new gaming machine license in order to allow the harmful impacts of venuebased gambling to be considered.

To assess the social impact of individual venues effectively we need to know the spatial distribution of the venue's clientele - the people most directly impacted by the venue. However, surprisingly little is known about the extent and intensity of the 'catchments' of gambling venues - that is, the spatial coverage of the catchments and the proportion of residents within them who visit gambling venues. To date, only two studies have specifically investigated the geography of gambling venue catchments. KPMG Consulting (2000) asked survey respondents how far they travelled to the gambling venue on the last occasion they gambled on EGMs in Victoria, Australia. From this data, KPMG produced an averaged $2.5 \mathrm{~km}$ radial catchment for each venue. However, this estimate is problematic because it relies on the dubious assumptions that (a) respondents can reliably estimate their own 
travel distances (cf. Walmsley \& Jenkins, 1992) and b) the size and shape of catchments is identical across all venues. In contrast, Doran et al. (2007) performed a geocoded household survey that asked respondents to identify their preferred gambling venue. Network distance was calculated from each respondent's residence to his or her preferred gambling venue. This study found considerable variation in catchment radius, from over $14 \mathrm{~km}$ to less than $4 \mathrm{~km}$.

Both of these studies assumed that the catchment areas of venues are constant across all groups of visitors. However, there is reason to doubt this claim. Young et al. (2012) found that EGM gamblers and problem gamblers are more likely to visit venues closer to their homes than non-gamblers and nonproblem gamblers. If catchment sizes differ between groups of visitors, then a "one size fits all" approach to catchment estimation may not be appropriate for social impact assessment.

While the findings produced by geocoded population surveys are able to provide catchment information for use in social impact assessments, they may be prohibitively expensive to conduct. Of greater utility would be a predictive tool that could accurately estimate venue catchments for a range of venue sizes using secondary, freely available, data. Here the gravity modelling approach developed in retail and trade geography may be useful. In particular, the Huff model (1964) has been used for over four decades to probabilistically estimate the market areas of retail outlets, and is still considered the best tool for this purpose in conjunction with contemporary Geographic Information Systems (GIS) (Huff \& McCallum, 2008).

Three studies have employed the Huff model to investigate gambling venue catchments.
Doran and Young (2010) developed a local measure of gambling accessibility using a Huff model at the city-scale. Their analysis was replicated for metropolitan Melbourne by Rintoul et al. (2012), who sought to demonstrate that gambling expenditure was associated with EGM accessibility and to identify localities where harm-minimisation efforts are most urgently needed. At the national scale, Markham et al. (2014) employed the Huff model to estimate the catchment areas of casinos in Australia. None of these studies empirically calibrated model parameters, instead using parameters selected a priori from the trade-area modelling literature. Despite the sound theoretical basis of Huff models, this is problematic because model accuracy is highly dependent on the parameters chosen. To date, no study has calibrated a trade-area model for gambling against venue visitation data, nor have the predictions of these models been compared to actual gambler behaviour. Consequently, the utility of the Huff model in a gambling context remains untested. To address this shortfall, we calibrated a Huff model to estimate gambling venue catchments using a large, geocoded postal survey. We posed three specific research questions:

(1) To what extent can Huff models predict the spatial distribution of gambling venue patrons?

(2) Which parsimonious configuration of model parameters provide the best model fit with observed visitation data?

(3) Does venue attractiveness vary between population subgroups?

We empirically assessed the suitability of the Huff model for explaining gambling venue visitation patterns using goodness-of-fit indices. Results of this calibrated model were compared with those from a previous 
normative study (Doran \& Young, 2010). We then compared Huff models for visitor subgroups to investigate differences in venue-choice behaviour.

\section{Study-area}

As part of a larger project, this paper builds on a trajectory of gambling research in the Northern Territory (NT) of Australia (Doran \& Young, 2010; e.g. Young, Lamb, \& Doran, 2009). The NT is notable for its relatively small population (229,711 in 2010), geographic remoteness, and relatively high proportion of Indigenous residents (30\%, compared to $3 \%$ in the rest of Australia). This study specifically focused on the three largest towns in the NT, which contain an estimated $63 \%$ of its population: Darwin $(107,430$ persons), Alice Springs (27,987 persons) and Katherine (10,104 persons) (Australian Bureau of Statistics, 2011). EGMs in the NT are concentrated in these three towns, which hosted $88 \%$ of the jurisdiction's EGMs ( $\mathrm{n}=$ 1,798 ) in June 2010 , but just $63 \%$ of its population. While $46 \%$ of the EGMs in these towns are located in casinos in Darwin and Alice Springs (833 EGMs), gambling opportunities are dispersed across the study site, with EGMs available in 26 clubs (612 EGMs) and 36 hotels (353 EGMs). Clubs, such as sporting or returned servicepersons clubs, are not-for-profit entities restricted to a cap of 45 EGMs per venue. Hotels or pubs are private businesses capped at 10 EGMs per venue.

\section{Data and Methods}

We used the Huff model to estimate gambling venue catchments. The Huff model is a form of spatial interaction model, which seeks to describe in a spatially explicit manner flows of people across space to a fixed set of locations in order to access goods or services. The Huff model takes the form:

$$
P_{i j}=k \cdot o_{j}^{\gamma} \frac{\left(\prod_{l} a_{i l}^{\alpha_{l}}\right) \cdot f\left(d_{i j}, \beta\right)}{\sum_{i}\left[\left(\prod_{l} a_{i l}^{\alpha_{l}}\right) \cdot f\left(d_{i j}, \beta\right)\right]}
$$

where $P_{i j}$ is the probability of residents at origin $\mathrm{j}$ interacting with destination $i$; $o$ is the population of origin $j$; $a_{i l}$ is the $l$ th variable describing the attractiveness of destination $i$; $f$ is a function of the distance between origin $j$ and destination $i$; and $k, \gamma, \alpha_{l}$, and are parameters to be empirically estimated. When fit using actual flow data, these estimated parameters can be interpreted in a similar manner to the output of ordinary linear regression, with the outcome variable representing the estimated probability of interaction between a source and destination, conditional on a set of predictor variable values. Like ordinary linear regression, the Huff model can be used to describe patterns in a data set, test hypotheses or make predictions.

The Huff model calibration process requires both population-level gambling venue visitation data and venue-level attractiveness data (see Figure 1). We used postal methods to collect venue visitation data and compiled venue-level attractiveness variables from secondary data sources. We then calculated distances between respondent residential locations and gambling venues and aggregated survey responses using two different spatial zoning schemes. We assessed a series of Huff model configurations using different distance measures, combinations of attractiveness variables, and distance decay functions to determine which of a variety of parameter configurations to include in our catchment model. Finally, using the model configuration identified as most appropriate, we estimated venue catchments for a series of patron 
subgroups likely to be of interest to gambling addresses across the peri-urban fringes of regulators.

Alice Springs and Darwin, to which Australia

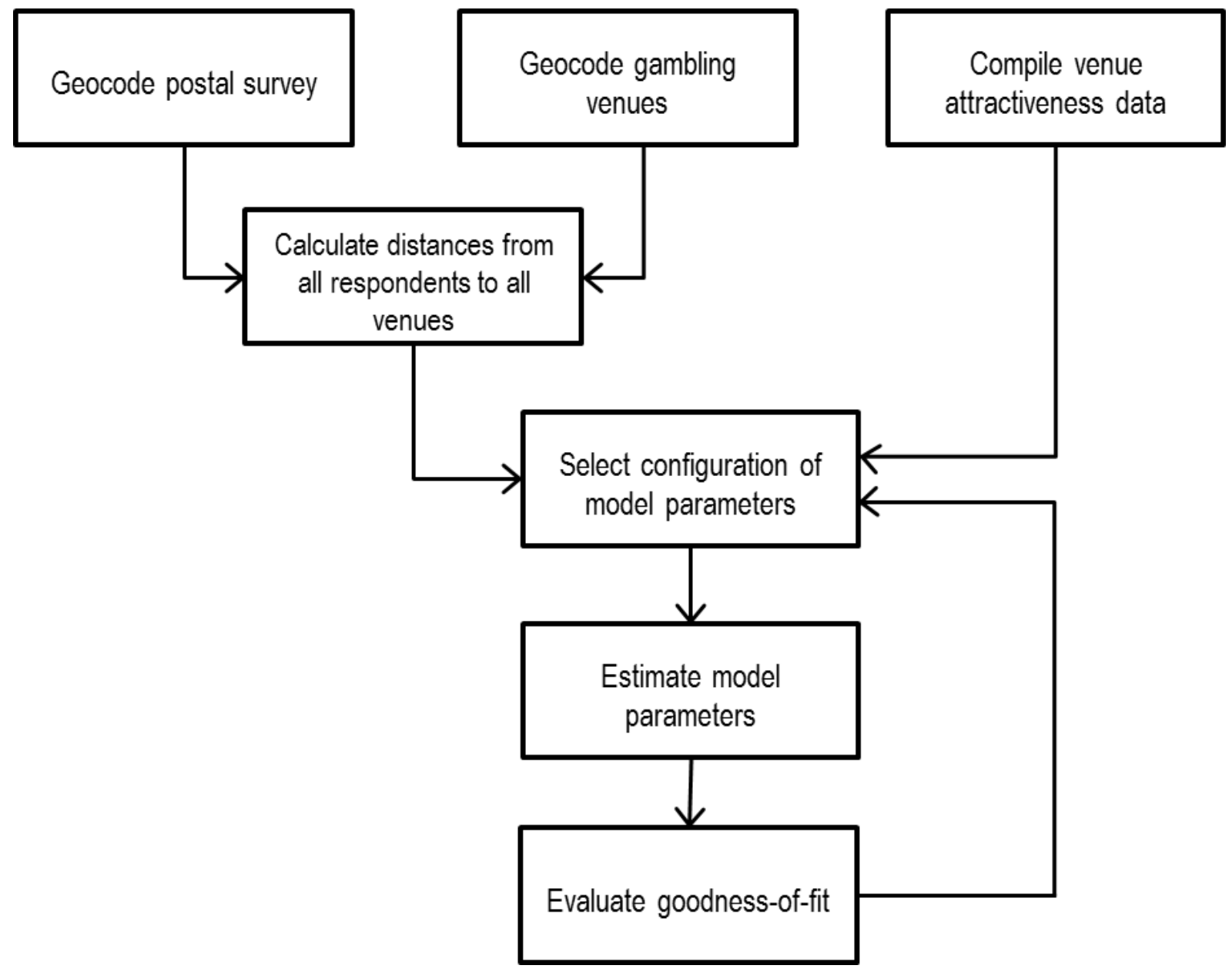

Figure 1. Huff model calibration process diagram.

\section{Visitation data}

Using the geocoded national address file (or G-NAF (PSMA Australia, 2010)) as a sample frame, we conducted a postal survey of 46,263 addresses in Darwin, Katherine and Alice Springs. The G-NAF is an authoritative database of verified geocoded street addresses for Australia, collated from various government agency databases including those of the Australian Electoral Commission and Australia Post. We mailed questionnaires to all G-NAF addresses to which Australia Post would deliver unsolicited mail and which were zoned as residential. To extend our spatial coverage, we selected 2,300
Post does not deliver mail, for hand delivery of questionnaires. The authors drove to the selected addresses and pegged questionnaires with reply-paid envelopes to gates and fences. In Alice Springs, 300 handdeliveries were conducted within a $15 \mathrm{~km}$ radius of the CBD. In the Darwin peri-urban fringe, we used a spatially stratified cluster sample design to select 2,000 out of a potential 7,000 addresses in a band $20 \mathrm{~km}$ to $40 \mathrm{~km}$ from Darwin's Central Business District. For the purpose of sampling, we divided this spatial band into four concentric tracts, each $5 \mathrm{~km}$ wide and selected 500 addresses for hand delivery in each tract, grouped into several contiguous blocks. The questionnaires were mailed between April 
and August 2010 and hand delivered to Alice Springs and Darwin in July and September 2010 respectively. Any household member aged eighteen or older was eligible to respond, and return of the survey implied consent. The Human Research Ethics Committee of Charles Darwin University granted approval to conduct the study (protocol no. Ho9048).

The questionnaire asked which gambling venues the respondent had visited in the last month. Respondents selected their most frequently visited venue from a list of all EGM venues in or proximate to their town of residence. Participants were asked to report the number of times they had visited this venue in the last month, whether they participated in EGM gambling on their last visit, and to complete the Problem Gambling Severity Index (PGSI) for the last twelvemonths (Ferris \& Wynne, 2001). We used the PGSI as our measure of gambling-related harm as it is a clinically-validated scale used to estimate problem-gambling risk in the general population (Ferris \& Wynne, 2001; Neal, Delfabbro, \& O’Neil, 2005). The PGSI is a nine-question screen, with responses ranging on an ordinal scale from 0-27, routinely classified into groups having no risk (PGSI 0), low risk (PGSI 1-2), moderate risk (PGSI 3-7) or high risk (PGSI $8+$ ) of being a problem-gambler. A unique identifier that referenced the respondent's G-NAF record was also included on the questionnaire, enabling survey returns to be precisely geocoded.

\section{Venue attractiveness data}

For each venue, we obtained data on type of gaming license (i.e. hotel, club or casino), number of EGMs licensed to the venue on June 30 2010, and street addresses from the Northern Territory Department of Justice.
We manually geocoded venue addresses using Google Maps. We also selected several venue-level spatial variables relevant to venue visitation behaviour: proximity to centres of community congregation, distance from the Central Business District (CBD) as measured by distance to the general post office, participation in the tourism-oriented night-time economy, and proximity to the ocean. In particular, proximity to centres of community congregation has been suggested as an important predictor of gambling catchments as these venues have potential to tap the pre-existing activity spaces of large numbers of residents (e.g. Doran et al., 2007). We used road network distance to closest supermarket as a proxy measure of proximity to areas of community congregation and obtained supermarket location data from the websites of the two supermarket operators who collectively supply $75 \%$ of the grocery market (Australian Competition and Consumer Commission, 2008). We included distance from the CBD in this study due to the differences in EGM gambling markets between suburban venues and inner city venues (e.g. Young et al., 2009). We measured distance to the CBD by proxy as road network distance to the general post office. Proximity to the ocean was included in this model due to our observation during exploratory data analysis that venues located within $100 \mathrm{~m}$ of the ocean had more patrons than might be otherwise expected, an observation consistent with previous research conducted at a courser spatial scale (Wardle, Keily, Astbury, \& Reith, 2012). We defined venues as proximate to the ocean if they were located within $100 \mathrm{~m}$ of the coastal boundary.

While Rintoul et al. (2012) used gambling expenditure as the measure of venue attractiveness in their Huff model, we considered this inappropriate for a study 
concerned with estimating general visitation. Specifically, because visitation is a necessary condition for EGM gambling, explanation of visitation behaviour based on gambling expenditure is temporally inconsistent and violates model assumptions.

Distance calculations, spatial aggregation and parameter estimation

The Huff model can be extended to incorporate different measures of distance. We calculated the distance between each survey respondent and EGM venue using Euclidian, Manhattan and road network measures of distance (in kilometres). We excluded respondents who did not report visiting any venue in the past month. We aggregated individual responses into Mesh Blocks and census collector districts in order to test which level of spatial aggregation would provide the best model fit without biasing parameter estimates. The distance from each zone to each venue for the three distance measures was estimated by calculating distances at the household level and then taking the median in each zone (Batty and Sikdar 1982).

Mesh blocks are a micro-level geographical unit, with a size of 20-50 dwellings in residential areas. Mesh blocks were chosen as the origin zones in this study as their relatively small size minimizes the effect of the modifiable areal unit problem (MAUP). Census collector districts correspond to the area assigned on census night to a single census officer, and contain an average of seven mesh blocks in our study area. In our case, the MAUP - that is, bias resulting from the use of arbitrary administrative zones to aggregate respondents - might make it difficult to know whether parameter estimates are the result of actual travel behavior or just the choice of zoning system
(Openshaw, 1977). Although more susceptible to the effects of the MAUP, collector districts were included in this study in order to increase the number of survey respondents per unit. When comparing systems of spatial aggregation at different scales that increasing average zone size will generally increase measures of model performance (Batty \& Sikdar, 1984). However, this does not necessarily mean that a better estimate of visitation behaviour has been derived. Rather, because larger zones will contain more responses, aggregation ameliorates the 'small numbers problem' whereby visitation patterns in zones with few respondents appears to be increasingly random (Batty \& Sikdar, 1982). Consequently, we compared the parameter estimates at different levels of spatial aggregation to see if the MAUP resulted in model bias.

We employed a composite measure of venue attractiveness following the observation in the retail trade-area literature that composite measures are more accurate predictors of shopping behaviour than centre size alone (Gautschi, 1981). Our measure included license type, number of EGMs, logarithmtransformed distance to supermarket, logarithm-transformed distance to CBD, having ocean views, and being a touristoriented inner-city bar. License type, ocean views and inner-city bar variables were coded as integer variables taking the values of one for false and two for true. We calibrated our model against a matrix of respondents most frequently visited venue, coded one if the venue was the preferred venue or zero if not.

Parameters were estimated using maximum likelihood methods using the $\mathrm{R}$ software package (R Development Core Team, 2012). We maximised the log-likelihood equation derived by Fotheringham and O'Kelly (1989) 
and computed confidence intervals for estimated parameters from the covariance matrix obtained by inverting the optimised hessian matrix. Goodness-of-fit was calculated using the R2 and Standardised Root Mean Square Error (SRMSE) metrics suggested by Fotheringham and O'Kelly (1989) and Thorsen and Gitlesen's (1998) Relative Number of Wrong Predictions (RNWP).

\section{Model selection}

In order to investigate which configuration of variables, distance measures, distance decay functions and venue-type subsets are most useful for predicting EGM gambling catchments we tested ten different models (see Table 1). Specifically, Model 1 was set to match the parameters used in the study published by Doran and Young (2010). Model 2 used the same configuration of variables, but allowed parameters to be estimated from the survey data. Subsequent models 3-9 were based on this configuration but each varied a single configuration option. Model 3 tested the effect of the removal of attractiveness index entirely. Model 4 introduced a composite attractiveness index. Model 5 modified Model 2 by aggregating responses to census collector districts. Models 6 and 7 tested the effect of using network and Manhattan measures of distance respectively. Model 8 used an exponential distance decay function in place of the power function. Model 9 included casinos and their visitors in the specification. Finally, in Model 10, we combined the best-fitting variants of the previously tested models.

\section{Comparison of visitor subgroups}

We re-estimated the best fitting Huff model configuration (Model 10) using visitation data for different subgroups of venue visitors. The subgroups of interest included:

(1) EGM gamblers (respondents who participated in EGM gambling on their last visit to their most frequently visited venue)

(2) non-gamblers (respondents who did not gamble on their last visit)

(3) moderate- to high-risk visitors (respondents with a PGSI score of three or more)

(4) non-problem gamblers (respondents with a PGSI score of zero)

(5) walkers (respondents who travelled on foot)

(6) frequent visitors (respondents who visited their most frequently visited venue four or more times in the last month)

(7) infrequent visitors (respondents who visited their most frequently visited venue once in the last month)

We selected these subgroups based on their relevance to regulators and our untested hypothesis that they would illustrate divergent travel behaviours. We mapped the estimated catchments for visitors from these different subgroups using kernel density estimation with a bandwidth of 500m, using ArcGIS 9.3 (ESRI, 2010). We selected three well-known venues in central and northern Darwin to map based on their diversity in terms of locational, licensing and patron characteristics. These three venues were chosen from all the venues in the study area ( $n=64$ ) for illustrative rather than analytic purposes and are indicative of the variation among venues. 


\section{Results}

\section{Survey respondents}

We received 7,040 survey responses (14.5\% response rate), with a median of 6 responses per Mesh Block (IQR $=4-8)$ or 32 responses per census collector district (IQR $=23-44$ ). Because all addresses in the sample frame were already geocoded, we achieved a $100 \%$ geocoding match rate. There was little evidence of spatial clustering of response rates at the Mesh Block level (Moran's I = o.003).

As is typical for surveys of this kind, the sample was older (mean age $=48.9$ ) and contained a higher proportion of women (61.8\%, $\mathrm{n}=4,292)$ compared to the population of the same area in the 2006 Census of Population and Housing (median age $=30-44$, proportion of women $=48.5 \%$ ). The majority of respondents were residents of Darwin $(77.3 \%, \mathrm{n}=5442)$, with the remaining respondents residing in Alice Springs (19.8\%, $\mathrm{n}=1393)$ and Katherine (2.9\%, $\mathrm{n}=205)$. Among the $71.1 \%(n=4,857)$ of respondents who had visited an EGM venue in the last month, 20.9\% ( $\mathrm{n}=1$,013) gambled on EGMs during their last visit. In terms of gamblingrelated harm $4.6 \%(\mathrm{n}=324)$ were at moderate risk (PGSI 3-7) and a further 2.0\% ( $\mathrm{n}=143$ ) were at high risk (PGSI 8+) of problem gambling. While this is a substantially higher level of gambling-related harm than that found in the most recent NT prevalence survey (0.64\% PGSI 8+, Young et al., 2006), this may be accounted for by incommensurate survey methods and relatively large standard errors.

A more detailed summary of survey responses is provided in the online supporting material.

\section{Model selection}

The Huff model was able to explain aggregate community-venue visitation patterns to a moderate degree (see Table 1). The best fitting model (Model 10, R2 $=0.64$ ) aggregated responses to the census collection district level, used a Euclidian distance measure, a power distance decay function, included casino venues, and included a variety of situational and licensing attractiveness variables. This represents a substantial improvement in explanatory power when compared to the use of Doran and Young's (2010) parameters (Model 1, R2 $=0.23$ ). Even when retaining Doran and Young's model configuration but empirically estimating parameters a substantial improvement in model fit was achieved (Model 2, R2 = 0.31).

In terms of individual attractiveness indices, the number of EGMs at a venue was a useful predictor of venue attractiveness. Venues with 45 EGMs were estimated to be 6.5 times as attractive as venues with a single EGM when number of EGMs was used as the only attractiveness variable (Model 2). The introduction of other licensing and situational variables increased the estimated magnitude of this relationship, with venues with 45 EGMs estimated to be 14.9 times as attractive as venues with a single EGM, holding other attractiveness variables constant (Model 4). As the EGM $\alpha$ is estimated to be less than 1.0 in all cases, the attractiveness of a venue does not increase linearly with addition of new EGMs as assumed by previous studies (Model 1).

The use of a multivariate attractiveness index provided a moderate improvement in model fit (Model 4, R2 = 0.35). Distance to the CBD, proximity to the ocean and being part of the inner-city night-time economy were all 
Table 1. Huff model parameter estimates for different configurations of parameters.

\begin{tabular}{|c|c|c|c|c|c|c|c|c|c|c|}
\hline & Model 1 & Model 2 & Model 3 & Model 4 & Model 5 & Model 6 & Model 7 & Model 8 & Model 9 & Model 10 \\
\hline Include casinos & No & No & No & No & No & No & No & No & Yes & Yes \\
\hline Zone & Mesh blocks & Mesh blocks & Mesh blocks & Mesh blocks & CCDs & Mesh blocks & Mesh blocks & Mesh blocks & Mesh blocks & CDs \\
\hline Distance type & Euclidian & Euclidean & Euclidean & Euclidean & Euclidean & Network & Manhattan & Euclidean & Euclidean & Euclidean \\
\hline Decay function & Power & Power & Power & Power & Power & Power & Power & Exponential & Power & Power \\
\hline$\beta$ & 1.5 & $1.09(1.06,1.12)$ & $1.14(1.11,1.17)$ & $1.08(1.05,1.11)$ & $1.13(1.09,1.16)$ & $1.17(1.13,1.20)$ & $1.07(1.04,1.10)$ & $0.17(0.17,0.18)$ & $1.02(0.99,1.05)$ & $1.02(0.99,1.05)$ \\
\hline EGMs $\alpha$ & & $0.49(0.44,0.54)$ & & $0.68(0.61,0.76)$ & $0.48(0.43,0.53)$ & $0.47(0.42,0.52)$ & $0.50(0.45,0.55)$ & $0.56(0.51,0.61)$ & $0.52(0.48,0.57)$ & $0.71(0.64,0.77)$ \\
\hline Casino $\alpha$ & & & & & & & & & $0.73(0.47,0.99)$ & $-1.72(-2.14,-1.29)$ \\
\hline Club a & & & & $-0.00(-0.15,0.14)$ & & & & & & $-0.33(-0.46,-0.19)$ \\
\hline ln dist. to supermarket & et $\alpha$ & & & $-0.61(-0.84,-0.39)$ & & & & & & $-0.35(-0.56,-0.14)$ \\
\hline ln dist. to $\mathrm{CBD} \alpha$ & & & & $1.16(0.81,1.51)$ & & & & & & $0.70(0.41,1.00)$ \\
\hline $\operatorname{cean} \alpha$ & & & & $2.65(2.49,2.81)$ & & & & & & $1.76,2.06)$ \\
\hline Inner city bar $\alpha$ & & & & $0.89(0.63,1.15)$ & & & & & & $0.52(0.29,0.75)$ \\
\hline$\gamma$ & 1 & $1.00(0.95,1.05)$ & $1.00(0.95,1.05)$ & $1.00(0.94,1.05)$ & $1.00(0.94,1.06)$ & $1.00(0.95,1.05)$ & $1.05)$ & $1.00(0.95,1.05)$ & $1.00(0.95,1.05)$ & $9,1.00)$ \\
\hline$k$ & & $0.98(0.97,0.99)$ & $0.96(0.95,0.98)$ & $0.97(0.96,0.98)$ & $0.99(0.98,1.01)$ & $0.99(0.98,1.00)$ & $0.99(0.98,1.00)$ & $1.12(1.11,1.13)$ & $0.91(0.90,0.92)$ & $1.16(1.15,1.18)$ \\
\hline $\mathrm{R}^{2}$ & 0.23 & 0.31 & 0.27 & 0.35 & 0.55 & 0.31 & 0.31 & 0.28 & 0.36 & 0.64 \\
\hline SRMSE & 4.8 & 4. & 4.6 & 4.4 & 2.5 & 4.5 & 4.5 & 4.6 & 4.1 & 2.2 \\
\hline RNW & 1.3 & 1.4 & 1.4 & 1.3 & 1.0 & 1.4 & 1.4 & 1.5 & 1.3 & 0.8 \\
\hline$n$ respondents & 3907 & 3907 & 3907 & 3907 & 3907 & 3907 & 3907 & 3907 & 4977 & 4977 \\
\hline
\end{tabular}

Note: Cells shaded grey indicate changes to model selection compared to Model 1, which was derived from Doran and Young (Doran \& Young, 2010). 95\% confidence intervals for parameter estimates are indicated in parentheses. Bold cells indicate $95 \%$ confidence intervals that do not overlap zero for $\beta$ and $\alpha$ or one for $\gamma$ and $k$. No confidence intervals are indicated for Model 1 because these parameters were input based on previous research, instead of being estimated from the data. An odds ratio is obtained for a binary attractiveness variable $\alpha$ using the formula $2^{a}$. The attractiveness contribution of a continuous attractiveness variable $\alpha$ with value $x$ is obtained by the expression $x^{a}$. CCDs refer to census collector districts.

Table 2. Huff model parameter estimates for different subgroups of visitors.

\begin{tabular}{|c|c|c|c|c|c|c|c|c|}
\hline & Group A & Group B & Group C & Group D & Group E & Group F & Group G & Group H \\
\hline Subgroup & All visitors & EGM gamblers & Non-gamblers & PGSI $\geq 3$ & PGSI $=0$ & Walkers & Frequent visitors & Infrequent visitors \\
\hline & $1.02(0.99,1.05)$ & $1.18(1.09,1.27)$ & $1.01(0.97,1.05)$ & $1.16(1.04,1.28)$ & $1.06(1.03,1.10)$ & $1.71(1.58,1.84)$ & $1.13(1.07,1.19)$ & $0.93(0.88,0.99)$ \\
\hline EGMs $\alpha$ & $0.71(0.64,0.77)$ & $1.17(0.98,1.36)$ & $0.41(0.34,0.48)$ & $0.84(0.61,1.08)$ & $0.47(0.40,0.53)$ & $0.37(0.11,0.63)$ & $0.40(0.29,0.51)$ & $0.45(0.34,0.56)$ \\
\hline Casino $\alpha$ & $-1.72(-2.14,-1.29)$ & $-0.23(-1.37,0.92)$ & $-0.95(-1.46,-0.44)$ & $-0.59(-2.11,0.93)$ & $-0.59(-1.04,-0.14)$ & $-1.82(-3.66,0.03)$ & $0.05(-0.69,0.79)$ & $-0.31(-1.03,0.41)$ \\
\hline Club $a$ & $-0.33(-0.46,-0.19)$ & $0.12(-0.36,0.61)$ & $0.01(-0.14,0.16)$ & $-0.15(-0.71,0.41)$ & $-0.09(-0.23,0.04)$ & $-0.26(-0.75,0.23)$ & $0.25(0.01,0.49)$ & $-0.21(-0.43,0.01)$ \\
\hline ln dist. to supermarket $\alpha$ & $-0.35(-0.56,-0.14)$ & $-0.31(-0.96,0.34)$ & $-0.41(-0.67,-0.14)$ & $-0.52(-1.32,0.29)$ & $-0.00(-0.24,0.23)$ & $-1.59(-2.30,-0.87)$ & $-0.48(-0.87,-0.08)$ & $-0.37(-0.73,-0.00)$ \\
\hline $\ln$ dist. to $\mathrm{CBD} \alpha$ & $0.70(0.41,1.00)$ & $-0.26(-1.07,0.56)$ & $-0.47(-0.79,-0.15)$ & $-0.26(-1.31,0.80)$ & $-0.50(-0.79,-0.20)$ & -1.19 $(-2.11,-0.27)$ & $-0.17(-0.69,0.34)$ & $-0.58(-1.05,-0.10)$ \\
\hline Proximate to ocean $\alpha$ & $1.91(1.76,2.06)$ & $0.20(-0.33,0.74)$ & $2.36(2.19,2.53)$ & $1.21(0.59,1.83)$ & $1.99(1.84,2.15)$ & $2.20(1.64,2.77)$ & $1.56(1.28,1.84)$ & $2.16(1.92,2.40)$ \\
\hline Inner city bar $\alpha$ & $0.52(0.29,0.75)$ & $-0.18(-0.95,0.59)$ & $0.16(-0.10,0.41)$ & $-0.22(-1.13,0.69)$ & $-0.40(-0.65,-0.15)$ & $1.08(0.48,1.69)$ & $-0.26(-0.68,0.16)$ & $-1.15(-1.59,-0.71)$ \\
\hline$\gamma$ & $0.94(0.89,1.00)$ & $1.01(0.90,1.11)$ & $1.01(0.95,1.08)$ & $1.02(0.84,1.19)$ & $0.98(0.92,1.04)$ & $1.00(0.91,1.09)$ & $0.97(0.88,1.06)$ & $0.97(0.87,1.06)$ \\
\hline$k$ & $1.16(1.15,1.18)$ & $0.95(0.94,0.96)$ & $0.90(0.89,0.92)$ & $0.93(0.91,0.94)$ & $0.98(0.97,0.99)$ & $1.00(0.99,1.02)$ & $0.96(0.95,0.98)$ & $1.08(1.06,1.09)$ \\
\hline $\mathrm{R}^{2}$ & 0.64 & 0.72 & 0.53 & 0.44 & 0.60 & 0.65 & 0.46 & 0.50 \\
\hline SRMSE & 2.2 & 3.1 & 2.6 & 4.7 & 2.4 & 4.3 & 3.4 & 3.0 \\
\hline RNWP & 0.8 & 0.8 & 1.0 & 1.2 & 0.9 & 1.0 & 1.1 & 1.1 \\
\hline$n$ respondents & 4977 & 1001 & 3307 & 426 & 4097 & 492 & 1398 & 1603 \\
\hline
\end{tabular}

is obtained for a binary attractiveness variable $\alpha$ using the formula $2^{\alpha}$. The attractiveness contribution of a continuous attractiveness variable $\alpha$ with value $x$ is obtained by the expression $x^{\alpha}$. 
associated with increased venue attractiveness. Distance to a supermarket was inversely associated with venue attractiveness, meaning that respondents were more likely to visit venues close to busy shopping centres.

While the model provides an adequate fit to the data when aggregated at the Mesh Block level (Model 2, R2 $=0.31$ ), $\mathrm{CD}$ level aggregation dramatically improves model performance (Model 5, R2 = 0.55). Parameter estimates did not vary significantly when aggregating (see Models 2 and 5), indicating that in this case aggregation is unlikely to induce MAUP-related bias.

The use of network distance or Manhattan distance metrics had little impact on model fit (Models 6 \& 7). A distance decay power function better fitted the interaction data than an exponential function $(\mathrm{R} 2=0.28$, Model 8), an unusual result for intra-urban interactions (Fotheringham \& O'Kelly, 1989).

The inclusion of casinos in the model improved overall model fit $(\mathrm{R} 2=0.36$, Model 9). Respondents visited casinos for reasons over and above the number of EGMs they house, with Model 9 estimating casinos to be 1.7 times as attractive as a hypothetical noncasino venue with the same number of EGMs. Investigation of Variance Inflation Factors (VIFs) among the attractiveness variables revealed substantial levels of covariance between the number of EGMs in a venue and the venue's status as a casino (but no other pairs of attractiveness variables). The collinearity between these variables results in changing parameter estimates between models and means that these estimates should be treated with caution.

\section{Comparison of visitor subgroups}

Estimated gambling venue catchments varied substantially across subgroups (see Table 2). Compared to all visitors (Group A, $\beta=1.02$ ), distance decay was higher among those who gambled on EGMs on their last visit to a venue (Group $B, \beta=1.18$ ), those at moderate or greater risk of problem gambling (Group $\mathrm{D}, \beta=1.16$ ), those who walked to a venue (Group F, $\beta=1.71$ ), and frequent visitors to a venue (Group $\mathrm{G}, \beta=1.13$ ).

Attractiveness parameter estimates were similarly variable among subgroups. Compared to all visitors (Group A, $\alpha=0.71$ ), the number of EGMs was a more important predictor of attractiveness for those who gambled on EGMs on their last visit to a venue (Group $B, a=1.17$ ) and a less important predictor of attractiveness for nongamblers (Group C, $\alpha=0.41$ ), non-problem gamblers (Group E, $\alpha=0.47$ ), those who walked to a venue (Group F, $\alpha=0.37$ ), and frequent venue visitors (Group $\mathrm{G}, \alpha=0.40$ ). Other attractiveness variable estimates also fluctuated between subgroups. For example, while proximity to the ocean was not a significant predictor of attractiveness for EGM gamblers, non-gamblers were 5.1 times more likely to visit venues with ocean views than those without (Group C, $\alpha=2.36$ ).

The venue catchment maps revealed stark differences in catchment size and intensity between venues. Among all venues in Darwin, the SKYCITY Casino (Figure 2) had the largest and most intense catchment for every subgroup of visitors, with the exception of those travelling on foot. Indeed, for all visitors (Group A) SKYCITY Casino's catchment covers the entire residential area of the town. In contrast, the Beachfront Hotel, Darwin, (Figure 3) had a moderate 

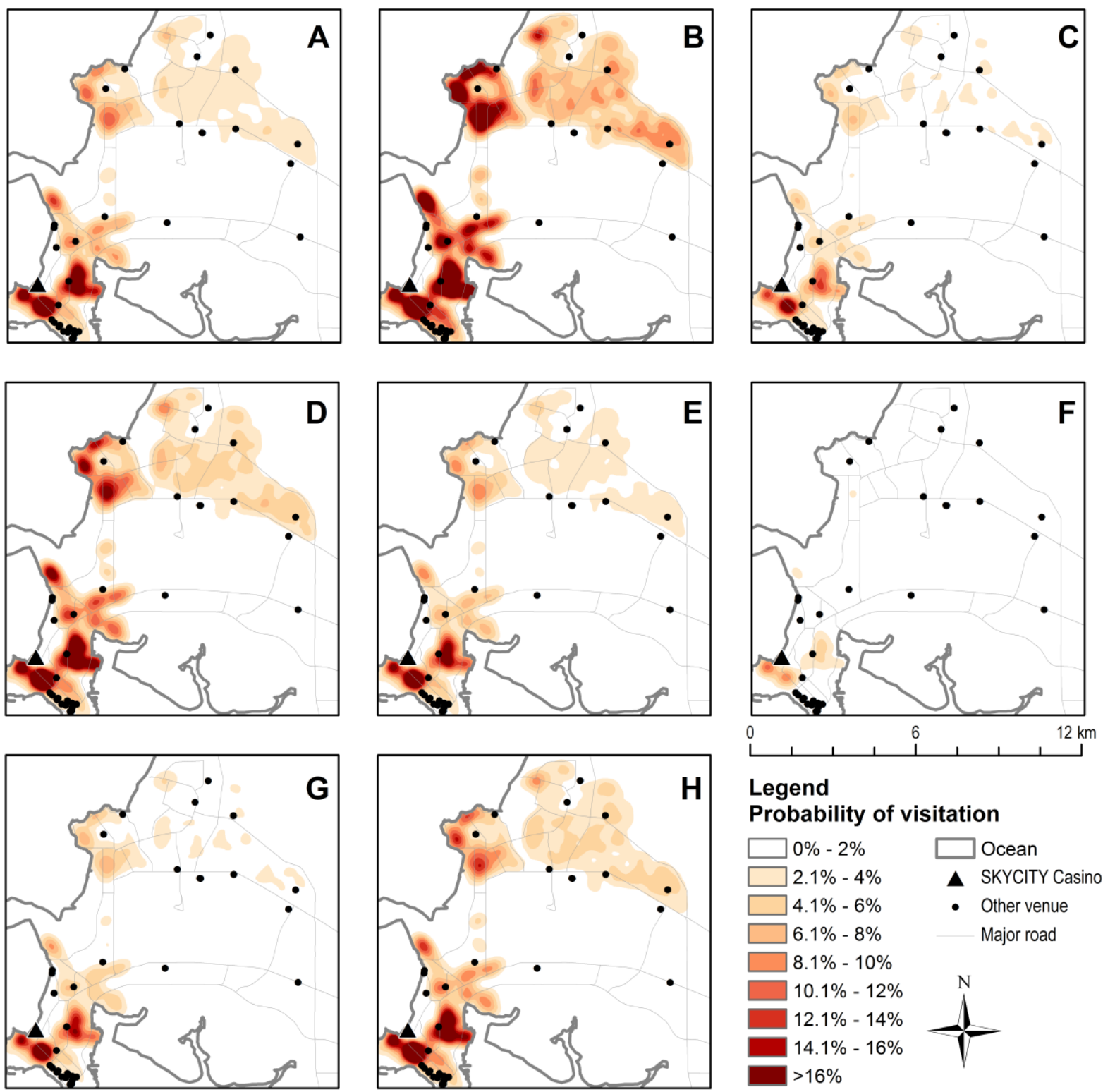

Figure 2. Estimated catchments of the SKYCITY Casino, Darwin, among different subgroups. $A=$ all visitors, $B=$ EGM gamblers, $C=$ non-gamblers, $D=$ moderate to high-risk visitors (PGSI $\geq 3$ ), $E=$ non-problem gamblers (PGSI $=0), F=$ walkers, $\mathrm{G}=$ frequent visitors ( $\geq 4$ visits per month), $\mathrm{H}=$ infrequent visitors ( 1 visit per month).

sized catchment for all visitors, covering only a few neighbouring suburbs. Similarly, the Casuarina Club's catchment in Darwin (Figure 4) was large yet localised to its surrounding region.

Different subgroups are attracted to different venues. Comparing the catchments for EGM gamblers with that for all visitors, SKYCITY Casino catchment (Figure 2) is largely unchanged in extent but substantially more intense, indicating a greater probability of EGM gamblers visiting this venue. In contrast, non-gamblers travel further to the Beachfront Hotel (Figure 3) than EGM gamblers. Like the casino, the Casuarina Club's catchment (Figure 4) for EGM gamblers is largely unchanged in size when compared to all visitors although it is slightly more intense.

\section{Discussion}

The calibration process substantially improved the explanatory power of the Huff 

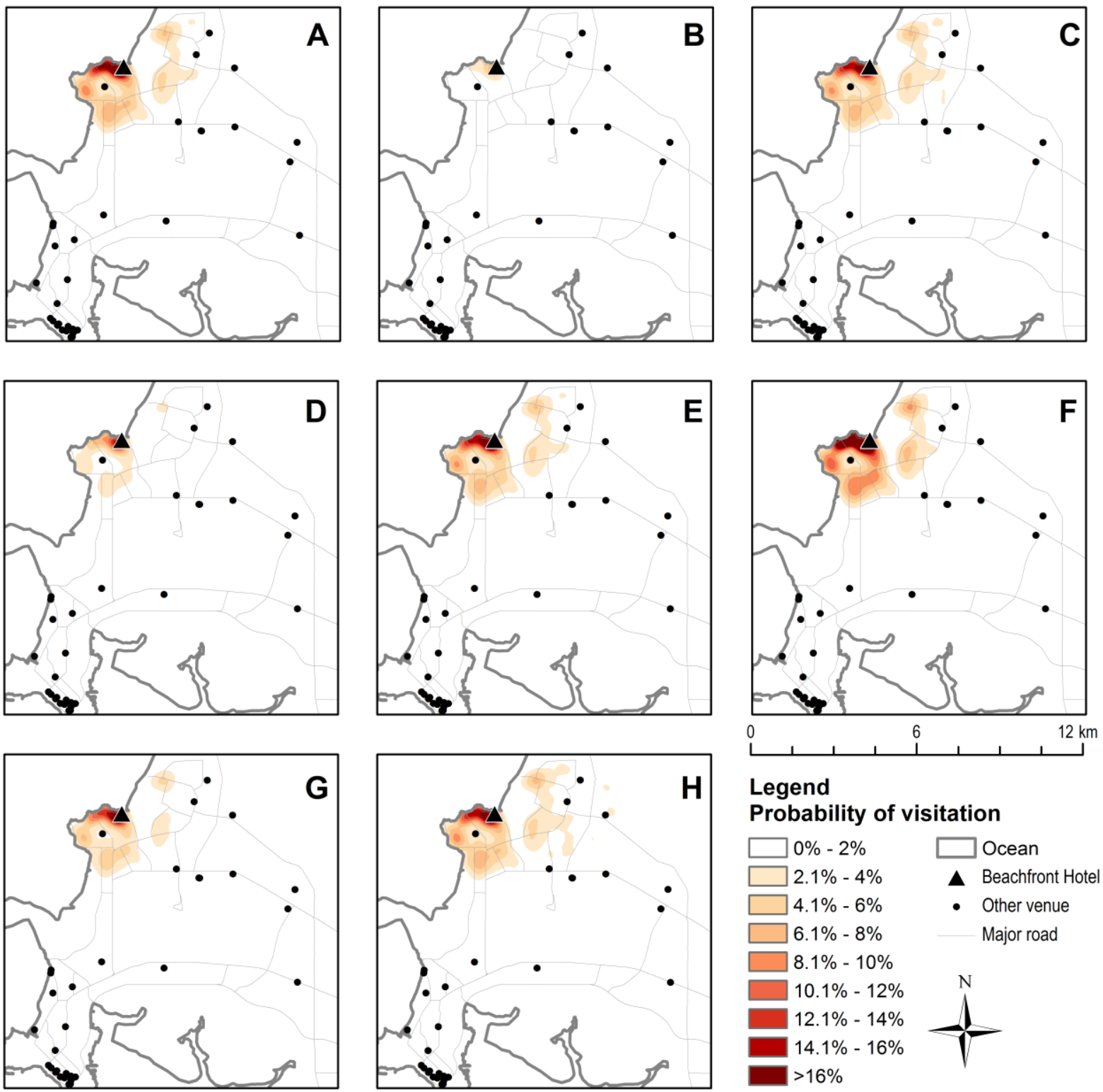

Figure 3. Estimated catchments of the Beachfront Hotel, Darwin, among different subgroups. $A=$ all visitors, $B=$ EGM gamblers, $C=$ Non-gamblers, $D=$ moderate to high risk visitors (PGSI $\geq 3$ ), $E=$ non-problem gamblers (PGSI $=0), F=$ Walkers, $\mathrm{G}=$ frequent visitors ( $\geq 4$ visits per month), $\mathrm{H}=$ Infrequent visitors (1 visit per month).

model, increasing the R2 from 0.23 to 0.64 . The estimated parameters may be usefully employed to predict gambling venue catchments in the NT and other jurisdictions with a similar configuration of EGM supply. This has potential benefits for social impact assessment because catchments can be more reliably estimated in places where surveys have not been conducted. Scenario-based models may be built in other jurisdictions such as Western Australia where EGMs have not yet been introduced outside of the local casino to model the spatial extent of potential social impacts of liberalising EGMs at the local level.

More specifically, we have confirmed the importance of various factors influencing venue choice that have previously only been assumed to be important. In particular, EGM numbers were important in explaining venue visitation behaviour for all subgroups. Indeed, for EGM gamblers, it was the only significant predictor of venue attractiveness. The fact that distance decay was particularly 

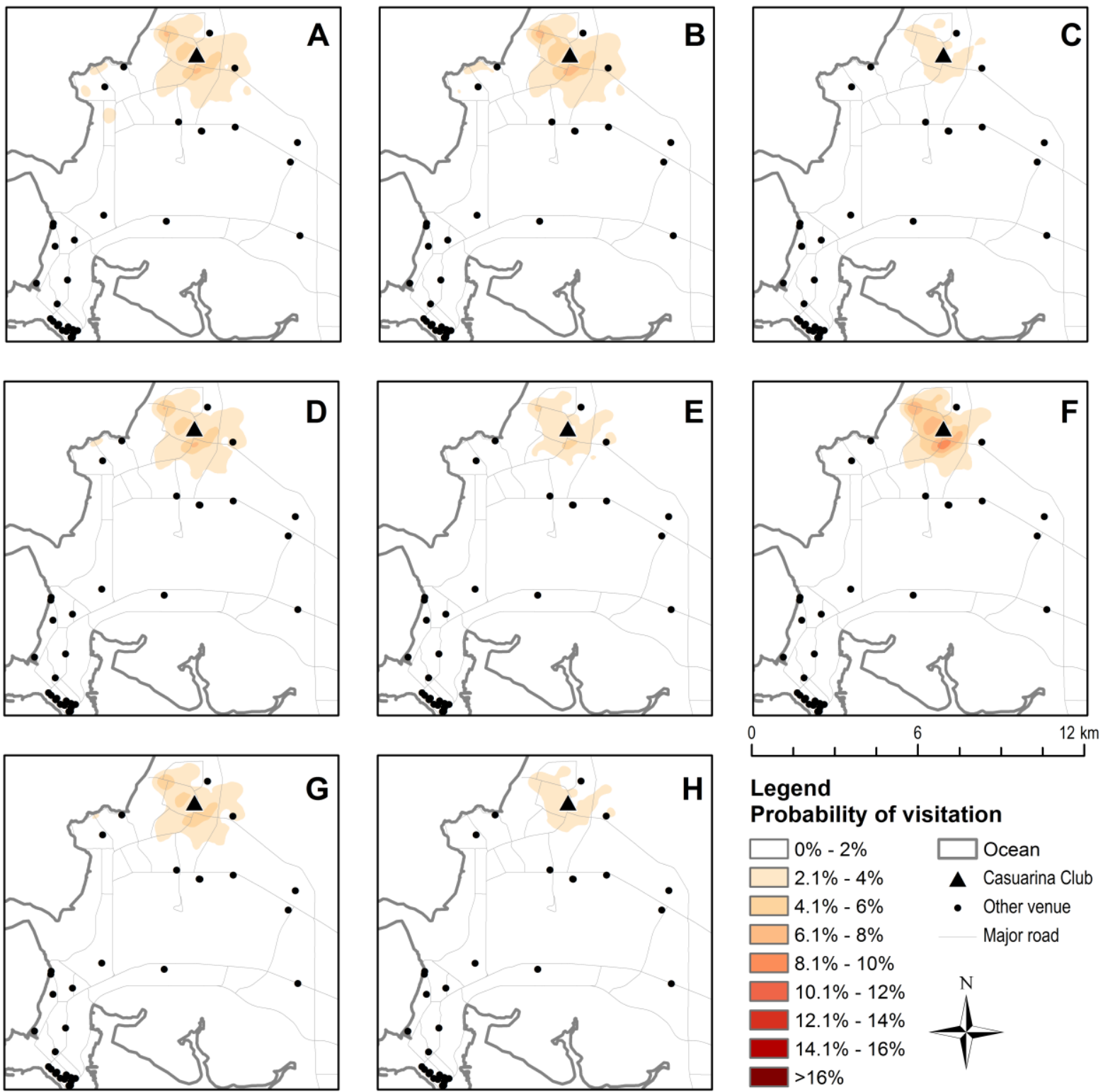

Figure 4. Estimated catchments of the Casuarina All Sports Club, Darwin, among different subgroups. $A=$ all visitors, $\mathrm{B}=\mathrm{EGM}$ gamblers, $\mathrm{C}=$ Non-gamblers, $\mathrm{D}=$ moderate to high risk visitors (PGSI $\geq 3$ ), $\mathrm{E}=$ non-problem gamblers (PGSI $=0), F=$ Walkers, $G=$ frequent visitors ( $\geq 4$ visits per month), $H=$ Infrequent visitors (1 visit per month).

important for EGM and problem gamblers indicates that accessibility is more important to these visitors than non-gamblers.

While previous research has shown that catchment sizes vary between venues (Doran et al., 2007), we have extended this result to show that catchment sizes vary between groups of visitors, even for the same venue. Furthermore, the direction of this effect is venue specific. Put another way, different population subgroups are attracted to different venues. There is no single set of venue-level variables that optimally represents the 'attractiveness' construct for all patrons. This implies that future studies should consider the subpopulation of interest carefully prior to selecting the composition of venue-attractiveness indices.

These findings have two important implications for research and regulation. First, the catchment considered should be appropriate to the specific preventative or 
harm-minimisation strategy under investigation. Interventions to reduce the public health impacts of gambling venues may need to consider catchments for nongamblers, gamblers and problem gamblers separately. For non-gamblers, strategies such a reduction in exposure or accessibility may be considered, while for gamblers and problem gamblers preventative and harmminimisation strategies respectively are needed. In terms of targeting interventions, different catchments need to be considered for different stages in a public health approach.

Second, the spatial extent of the social impacts of gambling venues should not be assessed without explicit consideration of local factors. The spatial configuration of EGM supply, the characteristics of venues, and the spatial distribution of their patrons are all factors in determining venue visitation behaviour. Spatial behavioural models such as the Huff model may need to be employed in such future assessments.

Our findings are subject to a number of caveats. First, whilst the use of a mail survey based on the G-NAF holds a number of key advantages with respect to accurately geocoding responses, this technique misses hard-to-reach and mobile sub-populations such as visitors from these towns' hinterlands, transient workers, and other groups unlikely to respond to residential surveys. Second, the relatively low response rate, although typical for surveys of this kind (Nakaya et al., 2007), means that vulnerable groups in the community may be underrepresented in our results. A better approach to data collection might involve venue exit surveys, performed in collaboration between industry, regulators and researchers. Third, the findings may not be generalizable beyond settings with dispersed gambling machines (e.g. Australia, New Zealand, most of Canada and several states of the USA).

Future research might usefully focus on replicating these methods and findings in different geographic contexts. Such studies might also investigate the community-level effects of gambling harm and employ the evidence presented here regarding the attractiveness of gambling venues to better assess the role of accessibility in mediating harm. Future problem gambling prevalence surveys could usefully employ modern address matching technologies and collect household-level spatial data to test these relationships in other contexts.

\section{Conclusions}

Our finding that catchments vary not only between venues, but also between different subgroups of visitors, has important public health implications. Some venues attract more vulnerable visitors than others. Our results suggest that harm minimisation measures could be better targeted if they consider specific combinations of gambling venues and visitor subgroups. Spatial modelling can provide decision support for regulators tasked with approving new license applications or for evaluating the impact of health interventions such as mandated reductions in EGM numbers.

Model calibration resulted in a substantial improvement in model fit relative to previously published studies. A calibrated Huff model is suitable for application in urban contexts as a regulatory tool for social impact assessment and harm minimisation. Our parameter estimates might usefully be applied to improve the identification of vulnerability hotspots in other locales. Given the trend toward gambling liberalisation throughout much of the developed world, the 
ability better understand the spatial relations between gambling venues and the communities that support them is essential.

\section{Acknowledgements}

This research was supported by the Community Benefit Fund of the Northern Territory Government; The Australian Research Council [Linkage Project LP0990584]; The Northern Territory Research and Innovation Board.

\section{References}

American Gaming Association, 2012. The State of the States: The AGA Survey of Casino Entertainment, 2012 ed. [online]. Washington: American Gaming Association. Available from: http://www.americangaming.org/files/aga/uploads/docs/sos/aga_sos_2012.pdf [Accessed 11 May 2012].

Australian Bureau of Statistics, 2011. Regional Population Growth, Australia, 2009-1O [online]. Canberra: Australian Bureau of Statistics, Cat. No. 3218.o. Available from: http://www.abs.gov.au/ausstats/abs@.nsf/mf/3218.o [Accessed 27 October 2012].

Australian Competition and Consumer Commission, 2008. Report of the ACCC inquiry into the competitiveness of retail prices for standard groceries - July 2008 [online]. Canberra: Commonwealth of Australia. Available from: http://www.acc.gov.au/system/files/Grocery\%2oinquiry\%2oreport\%20\%20July\%202008.pdf [Accessed 25 July 2013].

Batty, M. and Sikdar, P.K., 1982. Spatial aggregation in gravity models: 4. Generalisations and large-scale applications. Environment and Planning A, 14 (6), 795-822.

Batty, M. and Sikdar, P.K., 1984. Proximate aggregation-estimation of spatial interaction models. Environment and Planning A, 16 (4), 467-486.

Currie, S.R., Hodgins, D.C., Wang, J., el-Guebaly, N., Wynne, H., and Chen, S., 2006. Risk of harm among gamblers in the general population as a function of level of participation in gambling activities. Addiction, 101 (4), 570-580.

Doran, B., Marshall, D., and McMillen, J., 2007. A GIS-based Investigation of Gaming Venue Catchments. Transactions in GIS, 11 (4), 575-595.

Doran, B. and Young, M., 2010. Predicting the spatial distribution of gambling vulnerability: An application of gravity modeling using ABS Mesh Blocks. Applied Geography, 30 (1), 141-152.

Eadington, W.R., 1998. Casino gaming - origins, trends and impacts. In: K.J Meyer-Arendt and R. Hartmann, eds. Casino gambling in America: Origins, trends and impacts. New York: Cognizant Communication Corporation, 3-15.

ESRI, 2010. ArcGIS Desktop (9.3) [Computer Program]. Redlands, CA: Environmental Systems Research Institute.

Ferris, J. and Wynne, H., 2001. The Canadian Problem Gambling Index: Final Report [online]. Canadian Centre on Substance Abuse. Available from: http://www.cclat.ca/2003\%20and\%20earlier\%20CCSA\%20Documents/ccsa008805-2001.pdf [Accessed 30 August 2011].

Fotheringham, S. and O'Kelly, M.E., 1989. Spatial Interaction Models: Formulations and Applications. Dordrecht; Boston: Kluwer Academic Publishers.

Gautschi, D.A., 1981. Specification of Patronage Models for Retail Center Choice. Journal of Marketing Research, 18 (2), 162-174.

Huff, D., 1964. Defining and Estimating a Trading Area. The Journal of Marketing, 28 (3), 34-38. 
Huff, D. and McCallum, B., 2008. Calibrating the Huff Model Using ArcGIS Business Analyst [online]. Redlands, California: ESRI. Available from: http://www.esri.com/library/whitepapers/pdfs/calibrating-huff-model.pdf [Accessed 18 October 2011].

Korn, D.A. and Shaffer, H.J., 1999. Gambling and the Health of the Public: Adopting a Public Health Perspective. Journal of Gambling Studies, 15 (4), 289-365.

KPMG Consulting, 2000. Report of the 1999 Longitudinal Community Impact Study. Melbourne: Victorian Casino and Gaming Authority.

Markham, F., Doran, B., and Young, M., in press. Estimating the spatial extent of casino catchments in Australia using a trade-area model. Growth and Change.

Nakaya, T., Fotheringham, A.S., Hanaoka, K., Clarke, G., Ballas, D., and Yano, K., 2007. Combining microsimulation and spatial interaction models for retail location analysis. Journal of Geographical Systems, 9, 345-369.

Neal, P., Delfabbro, P.H., and O’Neil, M., 2005. Problem Gambling and Harm: Towards a National Definition [online]. Melbourne: Report prepared for the National Gambling Research Program Working Party. Available from: http://www.adelaide.edu.au/saces/gambling/publications/ProblemGamblingAndHa rmTowardNationalDefinition.pdf [Accessed 9 March 2012].

Office of Economic and Statistical Research, 2012. Australian Gambling Statistics, 28th Edition. Brisbane, Qld: Queensland Treasury and Trade.

Openshaw, S., 1977. Optimal zoning systems for spatial interaction models. Environment and Planning A, 9 (2), 169-184.

Productivity Commission, 1999. Australia’s Gambling Industries. Canberra: AusInfo, Report No. 10.

PSMA Australia, 2010. Product description: G-NAF version 1.11. Canberra: PSMA Australia.

$\mathrm{R}$ Development Core Team, 2012. $R$ : A language and environment for statistical computing. Vienna, Austria: R Foundation for Statistical Computing.

Rintoul, A.C., Livingstone, C., Mellor, A.P., and Jolley, D., 2013. Modelling vulnerability to gambling related harm: How disadvantage predicts gambling losses. Addiction Research \& Theory, 21 (4), 329-338.

Shaffer, H.J., Hall, M.N., and Bilt, J.V., 1999. Estimating the prevalence of disordered gambling behavior in the United States and Canada: A research synthesis. American Journal of Public Health, 89 (9), 1369-1375.

Storer, J., Abbott, M., and Stubbs, J., 2010. Access or adaptation? A meta-analysis of surveys of problem gambling prevalence in Australia and New Zealand with respect to concentration of electronic gaming machines. International Gambling Studies, 9 (3), $225-244$.

Thorsen, I. and Gitlesen, J.P., 1998. Empirical Evaluation of Alternative Model Specifications to Predict Commuting Flows. Journal of Regional Science, 38 (2), 273-292.

Walmsley, D.J. and Jenkins, J.M., 1992. Cognitive Distance: A Neglected lssue in Travel Behavior. Journal of Travel Research, 31 (1), $24-29$.

Wardle, H., Keily, R., Astbury, G., and Reith, G., 2012. 'Risky Places?': Mapping Gambling Machine Density and Socio-Economic Deprivation. Journal of Gambling Studies, advanced online publication.

Wilkinson, J., 1996. NSW and Gambling Revenue [online]. Sydney: NSW Parliamentary Library Research Service, Briefing paper No. 16/96. Available from: http://www.parliament.nsw.gov.au/prod/parlment/publications.nsf/o/deg20d27ac d8d788ca256ecfooo96910/\$FILE/16-96.pdf [Accessed 3 July 2012].

Young, M., Abu-Duhou, I., Creed, E., Morris, M., Stevens, M., and Tyler, W., 2006. Northern Territory Gambling Prevalence Survey 2005. Darwin: School for Social and Policy Research \& School of Health Sciences, Charles Darwin University.

Young, M., Lamb, D., and Doran, B., 2009. Mountains and molehills: A spatiotemporal analysis of poker machine expenditure patterns in the Northern Territory. Australian Geographer, 40 (3), 249-269. 
Young, M., Markham, F., and Doran, B., 2012. Too close to home? The relationships between residential distance to venue and gambling outcomes. International Gambling Studies, 12 (2), 257-273. 